\title{
Construction of Finite Element Segmentation Algorithm Model of Image
}

\author{
Li Han and Pengyuan Wang \\ College of Computer and Communication Engineering, Zhengzhou University of \\ Light Industry \\ Corresponding E-mail: 2070382673@qq.com
}

\begin{abstract}
Grading the fruit by using machine vision system, it is hoped by the computer to recognize and understand the image automatically, in order to achieve this objective, the key step is to capture the suitable fruit images so that fruit image information can be effectively decomposed. Therefore, the final result of decomposition is to get some of the characteristics of each image with its own motifs, such as borders, shape and so on. By using these primitives, you can match a certain pattern, so as to determine the quality of the fruit. In this paper,it takes the overview of the finite element segmentation as a starting point, combined with the interpretation of the numerical algorithm and FCM algorithm functional convergence of the sequence, relying on Mumford-Shah function model to investigate the generation of fruit image finite element model.
\end{abstract}

Keywords: Fruit image; Finite element segmentation; Arithmetic model

\section{Introduction}

Finite element method is based on the variational principle, which can make the problem of finding solution of differential equations of the required mathematical model boundary value problem, firstly convert to the corresponding variational problem, namely, functional extremum problem, then it can use split interpolation, turning the variable sub-problem to the common multivariate function problem, which can ultimately come down to solving a set of multivariate algebraic equations, thus numerical solution of boundary value problems can be solved and obtained.

By means of the semiautomatic finite element grid generation method, it can take the advantage of computer to do a lot of complex work, just by using manual work to complete the work that computer is not easy to achieve, thus, efficiency can be greatly improved. With the increasingly usage of the finite element method, as well as the outcome of high-performance computers, the flat automatic finite element grid generation method comes into being.

In order to approximate the anisotropic nature of the function sequence elements which can be fully applied to the finite element grid modeling, so as to improve the model positioning accuracy for fine texture edge, therefore, algorithm can adopt anisotropic properties with adaptive finite element grid, which also can use Minimal metafunction the previous iteration of finite element grid to have the adaptive adjustment. By adaptive finite element grid, it can provide the desired adaptive optimal triangulation structure, thereby it can improve the efficiency and responsiveness speed of the iterations. [1] Adopting quasi-Newton minimization algorithm to approximate the MS Function and get solved, it can make each iteration objective function value declined, so as to ensure the global convergence during the approximation process, so as to avoid local minima, thereby overcoming the non-posedness when it solves MS Functional approximation. In this paper, it summarized the features of various finite element methods, proposing a new 
finite element grid generation method,namely, forming a uniform grid search cover the flat area, through "filtering", forming the intermediate portions of the grid, then taking the shortest distance as principle to determine the uneven grid of fruit image boundary region near the boundary. This kind of algorithm only requires a few necessary input parameters, then it can automatically finish the fruit image finite element segmentation.

\section{Methods}

\subsection{Adaptive Adjustment of Grid}

In order to generate the adaptive geometric triangulation, we can use an automatic grid generation software package, namely, BL2D developed by INRIA. In this package, using the generated background grid to have adaptive adjustment for the outlook grid. The purpose of optimizing triangulation structure is to make the edge near the triangular element have higher anisotropy ratio, and the direction of triangle elements should be parallel to the edge, while the region that is far away from the edge, the triangular elements anisotropy ratio is approximate to 1 . However, due to the minimization process, the theoretical edge set is unknown, therefore, it is necessary to estimate the prospects grid obtained from the background grid. In BL2D, the outlook grid is got through a "estimator" so as to construct out from the background grid.[2] Meanwhile, estimator can be got according to the measurement of each point of background grid.Measurement can be decided by symmetric positive definite matrix $2 \times 2, \mathrm{~A}=\left(\begin{array}{ll}a & b \\ b & c\end{array}\right)$, which has three-factor. Among them, the original measurement refers coefficient of $(a, b, c)$ that can satisfy the equation $(\mathrm{x}, \mathrm{y})\left(\begin{array}{ll}a & b \\ b & c\end{array}\right)(\mathrm{x}, \mathrm{y})^{\mathrm{r}}=1$, where $(\mathrm{x}, \mathrm{y})$ can refer to all the points whose distance to the origin point distance is 1 . Since this equation can use the elliptic equation whose center is at the origin to represent, so when the ellipse rotation's angle is $\theta$,

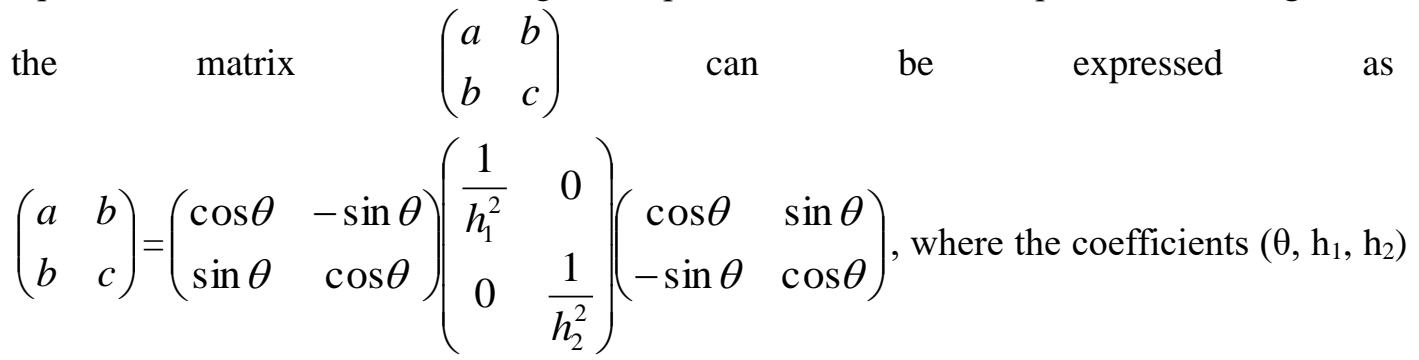
denote the rotation angle of the ellipse and the horizontal and vertical scale factor respectively. Therefore, before having adaptive grid, it just can be according to the function $\mathrm{u}$ to set the horizontal and vertical anisotropy values, namely, $\mathrm{h}_{1}, \mathrm{~h}_{2}$ and the rotation angle $\theta$. After giving the measurement of each point of background grid, it can construct a geometric shape such as the outlook grid of Delaunay triangulation. [3]

\subsection{Mumford-Shah Pan Function Model}

MS pan function model is a kind of energy function which includes homogeneity communicating area and the edges of objects through minimization, so that the original image can be divided into homogeneous regional connectivity, and make the discontinuous set of the segmentation function approximate to the edge of the target object, so as to achieve the effective segmentation for the fruit image. MS pan function model can be represented by the following formula.

$$
M S(u, K)=\iint_{\Omega \backslash K}|\nabla u|^{2} d x d y+a \iint_{\Omega \backslash K}|u-g|^{2} d x d y+\beta H^{1}(K)
$$


Among them, the region $\Omega$ is the bounded open set that can satisfy the boundary conditions of Lipschitz, while function $\mathrm{g}: \Omega \rightarrow[0,1]$ is the input fruit image, compact subset $\mathrm{K} \subset \bar{\Omega}$ is the edge formed by the discontinuity point set of $\mathrm{g}$, the function $\mathrm{u} \in \mathrm{C}^{\mathrm{l}}(\Omega \backslash \mathrm{K})$ is the smooth approximation on $\Omega \backslash \mathrm{K}$ for function $\mathrm{g}, \nabla \mathrm{u}$ is can be regarded as the gradient operator of function $\mathrm{u}, \mathrm{H}^{1}$ is the measurement of one-dimensional Hausdoff, $\alpha$ and $\beta$ are the two positive weight parameters. The three composed items of pan function MS (u, K), namely, $\iint_{\Omega \backslash K}|\nabla u|^{2} d x d y, \iint_{\Omega \backslash K}|u-g|^{2} d x d y$ and $\mathrm{H}^{1}(\mathrm{~K})$ can be respectively corresponding to the degree of smoothness, approximation degree and edge length constraint of $\mathrm{u}$ respectively. Among them, the smoothness constraints can ensure $u$ have sufficient smoothness on $\Omega \backslash \mathrm{K}$. Approximation constraints can ensure that $\mathrm{u}$ and $\mathrm{g}$ have small deviation on $\Omega \backslash \mathrm{K}$, the length constraint of edge can ensure that the length of the edge is limited, which can not fill the whole fruit image.

\subsection{FCM Algorithm}

FCM algorithm is an iterative optimization process. Assuming there us a fruit image, it's $n$ pixels can construct a fuzzy set $X=\left(x_{1}, x_{2}, \ldots, x_{n}\right)$, If each pixel is divided into $n$ fuzzy sets, each of the fuzzy subset has a class center vi, $i=0,1, \ldots, c-1$. If uik represents the $\mathrm{k}^{\text {th }}$ pixel 's the proximity of the $\mathrm{i}^{\text {th }}$ class, thus, it can get a matrix of all classes of the proximity to the all pixel, namely, $\mathrm{n} \times \mathrm{c}$ :

$$
\mathrm{U}=\left[\mathrm{u}_{\mathrm{ik}}\right], \mathrm{u}_{\mathrm{ik}} \in[0,1], \mathrm{i}=0,1, \ldots, \mathrm{c}-1, \mathrm{k}=0,1, \ldots, \mathrm{n}-1
$$

The proximity function of all the pixels of the $\mathrm{U} \mathrm{i}^{\text {th }}$ behavior to the class $\mathrm{i}$, the $\mathrm{k}^{\text {th }}$ column is the different types of proximity of the $\mathrm{k}^{\text {th }}$, meanwhile all columns should satisfy the following:

$$
\sum_{i=1}^{c} u_{i k}=1
$$

\section{The Introduction of FCM Algorithm}

\subsection{Introducing Fuzzy Weakening Operator}

By using fuzzy weakening operator $\partial(0<\partial<1)$, it can correct the membership function, enlarging the largest membership, suppressing the smaller membership, in this way, it can speed up the speed of algorithm optimization. Assuming vp is the largest membership category corresponding to the $\mathrm{i}^{\text {th }}$ pixel, thus, the principle of correcting of the membership function can be as follows:

$$
u_{i k}=\left\{\begin{array}{cc}
1-\partial \sum_{i \neq p} u_{i k} & k \neq p \\
\partial \times u_{i k} & k=p
\end{array}\right.
$$

The shearlets $\psi_{a, s, \mathrm{t}}$ emerge by dilation, shearing and translation of a function $\psi \in L_{2}\left(\square^{2}\right)$ as follows

$\psi_{a, s, t}(x):=a^{-3 / 4} \psi\left(A_{a}^{-1} S_{s}^{-1}(x-t)\right)=a^{-3 / 4} \psi\left(\left(\begin{array}{cc}\frac{1}{a} & -\frac{s}{a} \\ 0 & \frac{1}{\sqrt{a}}\end{array}\right)(x-t)\right)$ 
Where $A_{a}=\left(\begin{array}{cc}a & 0 \\ 0 & \sqrt{a}\end{array}\right)$ is a scaling (or dilation) matrix, $a \in \square^{+}, S_{s}=\left(\begin{array}{ll}1 & s \\ 0 & 1\end{array}\right)$ is a shear matrix, $s \in \square$ 。

We assume that $\hat{\psi}$ can be written as $\hat{\psi}\left(\omega_{1}, \omega_{2}\right)=\hat{\psi}_{1}\left(\omega_{1}\right) \hat{\psi}_{2}\left(\frac{\omega_{2}}{\omega_{1}}\right)$. Consequently, we obtain for the Fourier transform

$$
\hat{\psi}_{a, s, t}(\omega)=a^{3 / 4} e^{-2 \pi i\langle\omega, t} \hat{\psi}_{1}\left(a \omega_{1}\right) \hat{\psi}_{2}\left(a^{-1 / 2}\left(\frac{\omega_{2}}{\omega_{1}}+s\right)\right)
$$

The frequency plane is divided into four parts on the basis of different $\omega_{1}$ and $\omega_{2}$, that is horizontal cone $c^{h}$, vertical cone $c^{v}$, intersection(or the seam lines) of the two cones $c^{\times}$, low frequency part $c^{0}$, as shown in Figure 1 .

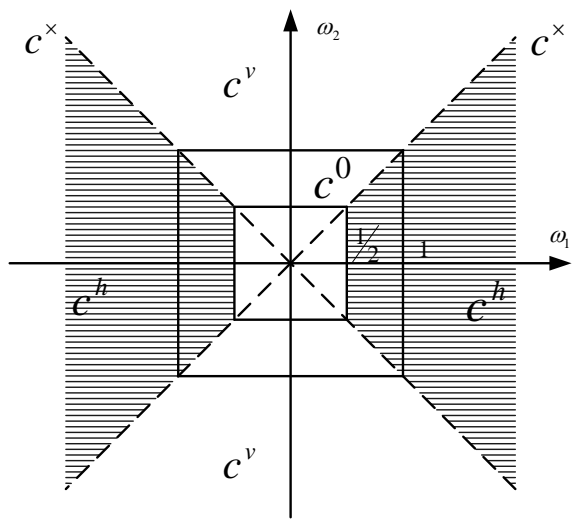

Figure 1. The Tiling of the Frequency Plane Induced by the Shearlet

In this paper, the adopted function can be shown in Figure 2, Among them, the abscissa is the reflection if the distance to the cluster center in each pixel $(1,1)$.

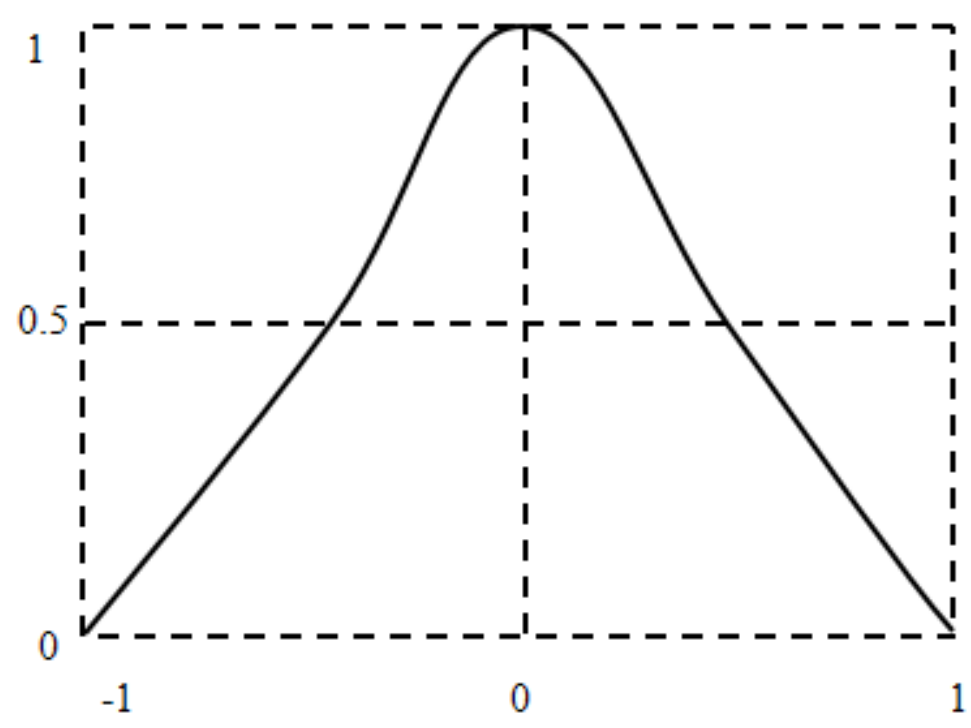

Figure 2. Fuzzy Weakening Operator $\partial$

Algorithm can adopt the above fuzzy weakening operator which also can absorb the competitive learning ideas, in this way, it not only can effectively reduce the membership 
of the study sample rate, which also can increase the learning rate of the membership samples as much as possible, so as to increase the role of membership sample on correcting the clustering center role, so that $\mathrm{V}$ can approximate clustering center quickly.

\subsection{The Generation of Finite Element Model of Image}

In ANSYS, the obtained fruit geometric model can use the above means to have segmentation for the finite element grid and get serial number of each finite elements as well as four vertex coordinates (assuming to adopt the tetrahedron unit), then based on the vertex coordinates of each tetrahedron unit to determine the coordinates of the center as well as the of coordinates of gravity of the tetrahedron, which can be shown in Figure 3. Assuming the coordinates of the four vertices are $\left(\mathrm{x}_{1}, \mathrm{y}_{1}, \mathrm{z}_{1}\right),\left(\mathrm{x}_{2}, \mathrm{y}_{2}, \mathrm{z}_{2}\right),\left(\mathrm{x}_{3}, \mathrm{y}_{3}, \mathrm{z}_{3}\right)$ and $\left(\mathrm{x}_{4}, \mathrm{y}_{4}, \mathrm{z}_{4}\right)$, taking into account that each tetrahedron element is equal mass distributed, so the coordinates of the gravity center can be calculated as follows:

$x_{0}=\frac{x_{1}+x_{2}+x_{3}+x_{4}}{4}, y_{0}=\frac{y_{1}+y_{2}+y_{3}+y_{4}}{4}, z_{0}=\frac{z_{1}+z_{2}+z_{3}+z_{4}}{4}$

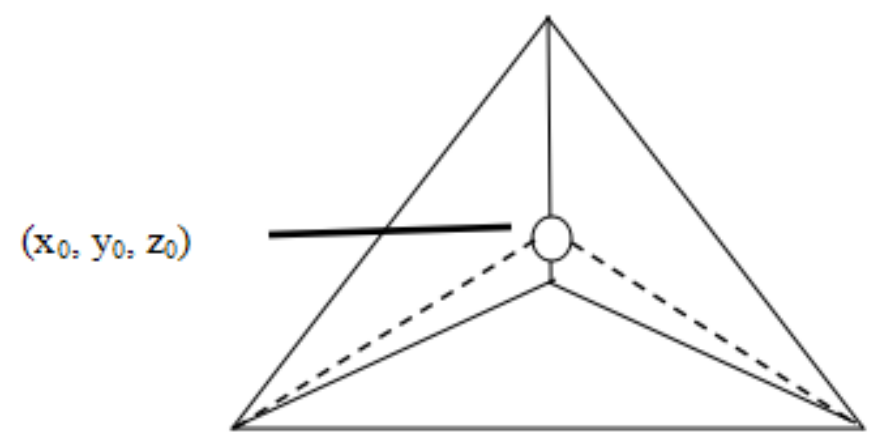

Figure 3. Schematic Graph of Coordinates of Tetrahedron Gravity Center

From the above we can see, just knowing the coordinates of any point in space, we can know what kind of organization will appear at this point. Therefore, we can determine the properties of the tetrahedron unit by the position of the gravity center of each organization tetrahedron unit, which can ultimately complete the establishment of the entire real finite element model.

\subsection{The Introduction of Kohonen Clustering Neural Network Sets the Initial Cluster as Center}

The determination of FCM initial clustering center can largely determine the convergence rate, if the initial clustering center is chosen from the clustering center that is close to the actual distance, the contrary iterative process can be greatly reduced, if the initial clustering center is selected get farther away from the actual clustering centers, it takes a long time to achieve convergence.[4] Kohonen Clustering Neural Network (KCN) is a kind of network with two layer structure, which is consisted by input layer and output layer and connected by a weight vector between the two layers. The structure can be shown in Figure 4: 


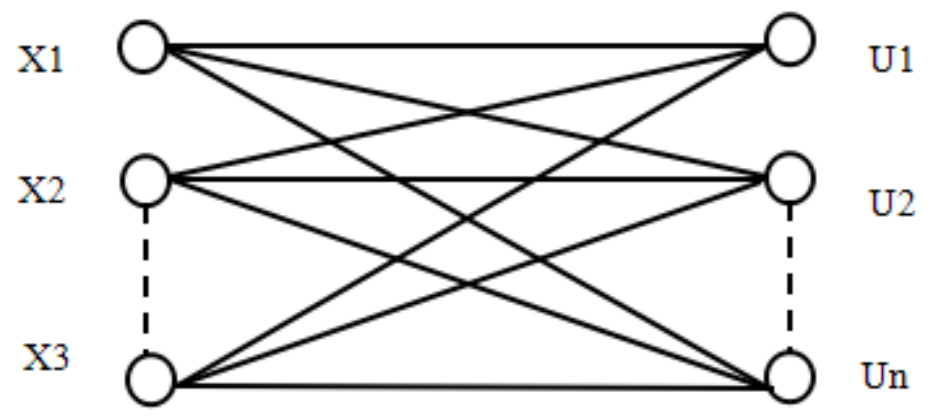

Figure 4. Kohonen Clustering Neural Network Structure

The steps of using KCN to have cluster analysis can be shown as follows:

(1) setting arbitrarily the initial cluster center $\mathrm{V}=\left(\mathrm{v}_{1}, \mathrm{v}_{2}, \ldots, \mathrm{v}_{\mathrm{c}}\right)$, as well as the convergence threshold as $\varepsilon$;

(2) calculating the Euclidean square distance of each pixel $x_{k}$ to each cluster center $\mathrm{d}^{2}{ }_{\mathrm{ik}}=\left(\mathrm{x}_{\mathrm{k}}-\mathrm{v}_{\mathrm{i}}\right)^{\mathrm{T}}\left(\mathrm{x}_{\mathrm{k}}-\mathrm{v}_{\mathrm{i}}\right)$;

(3) according to the following formula to correct d2ik m, so as to get the corresponding class when it gets minimum value.

$\mathrm{v}_{\mathrm{i}, \mathrm{t}+1}=\mathrm{v}_{\mathrm{i}, \mathrm{t}+1}+\mathrm{a}_{\mathrm{ik}, \mathrm{t}}\left(\mathrm{x}_{\mathrm{k}}-\mathrm{v}_{\mathrm{i}, \mathrm{t}}\right)$

Among them, $\mathrm{a}_{\mathrm{ik}, \mathrm{t}}$ is the learning coefficient of network, usually $0<\mathrm{a}_{\mathrm{ik}, \mathrm{t}}<1$, at the same time, it will be decreased when the number of iteration is increased.

(4) calculating $\mathrm{E}=\left\|V_{t}-V_{t+1}\right\|^{2}$, if $\mathrm{E}<\varepsilon$, then the training can be finished, otherwise, it will turn to (2).

(5) using the obtained $v$ acquired by network convergence, according to the principle of maximum degree of membership to have classification for each pixel.

As it can be seen from KCN algorithm flow, it is a hard clustering algorithm. In FCM algorithm, the effect of all image pixels on each class must be considered, while for $\mathrm{KCN}$, each fruit image pixel only affects its nearest class. In this way, the benefits of $\mathrm{KCN}$ is to get the network converges faster than that of FCM, but the disadvantage is the clustering accuracy will be lower than that of FCM. [5]

\section{Conclusion}

In order to complement the shortcomings of the two aspects, in this paper, it used KCN fruit image segmentation to have pre-classification, taking the clustering center by means of KCN as the initial cluster center of FCM,then used FCM to have further segmentation. Practice has proved that the result of doing so can ensure the accuracy of the segmentation, which also can greatly accelerate the efficiency of segmentation.

\section{References}

[1] D. C. Slaughter and R. C. Harrell, "Discriminating Fruit for Robotic Harvest Using color in Natural Outdoor Scenes", Trans of the ASAE, vol. 32, (1989), pp. 757-763.

[2] Z. Shuhai and T. Takahashi, "Studies on Autom Ation of Work in Orchards (part 1). Detection of Apple By Pattern Recognition”, Journal of the Japanese Society of Agricultural Machinery, vol. 58, (1998), pp. 9-16.

[3] N. Kondo, Y. Nishitsuji and P. P. Ling, "Visual Feed Back Guided Robotic Cherry to Mat to Harvesting", Trans of the ASAE, vol. 39, (1996), pp. 2331-2338.

[4] V. Caselles, R. Kimmel and G. Sapiro, "Geodesic active contours", Int'l Journal of Computer Vision, vol. 22, (1997), pp. 61-79.

[5] A. Yezzi, S. Kichenassamy, A. Kumar, P. Olver and A. Tannenbaum, "A geometric snake model for segmentation of medical imagery", IEEE Trans. on Medical Imaging, vol. 16, (1997), pp. 199-209.

[6] Sun Junding, Cui Jiangtao and W. Xiaoxing, "Color image retrieval method based on color and shape features", Chinese Journal of image and graphics, vol. 9, no. 7, (2004), pp. 820-827. 
[7] D. Chengqiang and F.Gang, "A multi feature integrated image retrieval based on content", Computer application, vol. 23, no. 7, (2003), pp. 100-102.

[8] W. Jie and Q. Zhengding, "Progress in research on fusion underlying content features in image retrieval", Chinese Journal of image and graphics, vol. 13, no. 2, (2008), pp. 189-197.

[9] M. Hu, "Visual pattern recognition by moment invariants", IRE Transactions on Information Theory, (8), (1962), pp. 179-187.

[10] H. Yiping, H.Xinsheng and L. Xiaolei, “A new invariant of gray variation”, Journal of Dalian Maritime University, vol. 34, no. 4, (2009), pp. 23-27.

\section{Author}

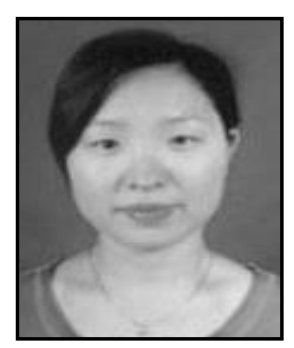

Li Han, received the M.E. degree in communication and information systems from Huazhong University of Science and Technology, Wuhan, China, in 2005.She is currently a lecturer of the College of Computer and Communication Engineering, Zheng Zhou University of Light Industry. Her major research interests includes software engineering and compilation technology, Intelligent information processing, etc. Research direction: Computer analysis. Data mining. 
International Journal of Signal Processing, Image Processing and Pattern Recognition Vol. 9, No. 11, (2016) 